\title{
A Schematic Two Overlapping-Band Model for Superconducting Sulfur Hydrides: The Isotope Mass Exponent
}

\author{
R. M. Méndez-Moreno $(D$ \\ Departamento de Física, Facultad de Ciencias, Universidad Nacional Autónoma de México, Avenida Universidad 3000, \\ Ciudad de México 04510, Mexico \\ Correspondence should be addressed to R. M. Méndez-Moreno; rmmv@ciencias.unam.mx
}

Received 30 April 2019; Revised 27 June 2019; Accepted 1 July 2019; Published 10 October 2019

Academic Editor: Oleg Derzhko

Copyright (c) 2019 R. M. Méndez-Moreno. This is an open access article distributed under the Creative Commons Attribution License, which permits unrestricted use, distribution, and reproduction in any medium, provided the original work is properly cited.

\begin{abstract}
The high value of the isotope shift in sulfur hydrides supports a phonon-mediated pairing scenario of superconductivity for these high-temperature superconductors which are consistent with the Bardeen-Cooper-Schrieffer (BCS) framework. Knowing that a large electronic density of states enhances the critical temperature $\left(T_{c}\right)$, generalized Fermi surface topologies are used to increase it. A multicomponent model within the BCS framework is proposed in this work for sulfur hydride superconductors. This model is used to evaluate some properties of the $\mathrm{H}_{3} \mathrm{~S}$ superconductor. Strong and intermediate coupling effects are taken into account with the effective McMillan approximation, and the isotope coefficient is evaluated as a function of the coupling parameter as well as other relevant parameters of the model.
\end{abstract}

\section{Introduction}

Research on the possibility of room temperature superconductivity has been invigorated by the discovery of superconducting sulfur hydrides at very high pressure, about 155 gigapascals (GPa) $[1,2]$. Given that hydrogen has the smallest atomic mass, it was predicted that metallic hydrogen or hydrogen-rich compounds would be high- $T_{\mathrm{c}}$ superconductors [3-5]. Light hydrogen atoms provide highfrequency phonon modes and strong electron-phonon coupling due to the lack of an electronic core. It has been shown that $\mathrm{H}_{2} \mathrm{~S}$ is stable below $43 \mathrm{GPa}$, and at elevated pressures it decomposes into $\mathrm{H}_{3} \mathrm{~S}$ and $\mathrm{S}$ [6-8]. $\mathrm{H}_{3} \mathrm{~S}$ has been found to be stable up to at least $300 \mathrm{GPa}$ [9]. The superconducting temperature of $\mathrm{H}_{3} \mathrm{~S}$ at pressure of about $150 \mathrm{GPa}$ is as high as $203 \mathrm{~K}$. This superconductor has one of the highest transition temperatures obtained in high-pressure experiments reported up until now $[10,11]$.

The high value of the isotope exponent in this material shows evidence of a phonon-mediated pairing mechanism, consistent with the BCS framework. The fact that the critical temperature varies with the isotopic mass is the evidence of the interaction between the electrons and lattice vibrations [12-14]. These results are proof that the electron-phonon interaction is an important pairing mechanism in the sulfur hydrides [15-17]. The observation that the superconductivity in these superconductors shows strong sensitivity to the crystal lattice suggests the possibility of unconventional electron-phonon coupling. Phonon properties in sulfur hydride systems are actually responsible for changes in their properties [18].

Understanding the electronic structure at the Fermi level can give some useful clues to unravel the fundamental ingredients responsible for the high transition temperature. However, up to now, the underlying physical process remains unknown. In this context, it seems crucial to study new ideas that use simplified schematic models to isolate the mechanism(s) that generate high transition temperatures. It was known that a large value of the density of states (DOS) at Fermi level increases the critical temperature value. That is, $T_{c}$ can be enhanced when the Fermi level is at or close to the energy of van Hove's singularity which provides a peak in the DOS [19-22]. Hirsch and Scalapino signaled the possibility of enhancing the superconducting critical temperature with a 
two-dimensional structure when the Fermi level was near a singularity [23]. First-principle calculations based on the supercell method have been reported where the authors study the effect of doping $\mathrm{H}_{3} \mathrm{~S}$ on the behavior of its superconductivity [24]. They show that $T_{c}$ in $\mathrm{H}_{3} \mathrm{~S}$ can be enhanced by hole doping.

A schematic model with generalized Fermi surface topologies, which take into account a two-component system with the same dispersion relation, has been proposed by us to study cuprate superconductors in the weak and intermediate coupling region. The generalized Fermi surface, via band overlapping, is proposed as a way of increasing the density of states at the Fermi energy. This model is based on the idea that the $T_{c}$ is enhanced when the Fermi level lies at or close to the energy of a singularity in the DOS. When combined with the Cooper pair equation, this schematic model can be taken as a simple device to model the singular behavior in the density of states and to account for the higher $T_{c}$ values obtained by cuprate superconductors [25-27].

Pairing symmetry is an important element toward understanding the mechanism of high- $T_{c}$ superconductivity. Calculations with two- and three-dimensional models using the BCS formalism and order parameter with $s$-wave symmetry have been reported in superconducting $\mathrm{H}_{3} \mathrm{~S}$ [28]. It was suggested that the search for better superconductors should be in three-dimensional systems where the thermal fluctuations are less likely to reduce the observed $T_{c}$ [29].

The Eliashberg equations are strong-coupling extensions to the original BCS theory. Calculations with this theory can be found where they study the high $T_{c}$ in sulfur hydride as a result of the variability in the DOS within the band [30]. Many theoretical calculations based on Eliashberg theory for $\mathrm{H}_{3} \mathrm{~S}$ can be found in the literature and also in [31], where the authors also provide an optical spectroscopy study for this material and found spectroscopic evidence that the superconducting mechanism in $\mathrm{H}_{3} \mathrm{~S}$ is due to the electron-phonon interaction. It has been shown that $\mathrm{H}_{3} \mathrm{~S}$ is a highly optimized electron-phonon superconductor [31]. As it is known, McMillan numerically solved the Eliashberg nonlinear equations at finite temperature in order to obtain the critical temperature for strong coupled superconductors. He obtained a parametrization which relates the critical temperature to a small number of parameters [32]. This approach, valid for the electron-phonon coupling constant $\lambda<1.5$, was later modified by Allen and Dynes to include values in the strong coupling region up to $\lambda \simeq 2$ [33-35].

$\mathrm{H}_{3} \mathrm{~S}$ is a multiband metal [36]. It was found that the optical properties of $\mathrm{H}_{3} \mathrm{~S}$ are different from those in conventional superconductors which are in the dirty limit [37]. $\mathrm{H}_{3} \mathrm{~S}$ is in the clean, phonon-dominated regime because of the very large energy scale associated to its phonon [37]. The electronic structure of $\mathrm{H}_{3} \mathrm{~S}$ has several Fermi surface sheets in the pressure range where high-temperature superconductivity appears [38]. However, it was found that the contribution to the total DOS, at the Fermi level, mostly comes from two Fermi surface sheets [39]. Based on the electronic band structure obtained from this material and on the results mentioned in this paragraph, we propose to evaluate the properties of sulfur hydrides with the topology of generalized Fermi surfaces modeled with band overlapping. A multiband structure within the BCS framework is proposed. This model, which can be taken as a minimal singularity in the density of states, together with the BCS framework, predicts higher $T_{c}$ values given that the energy band overlapping increases the DOS near the Fermi level [40-42]. The experimental results support the fact that the pairing mechanism is via phonon exchange. For physical consistency, an important requirement introduced in our model is that the band overlapping parameter is not larger than the phonon energy, $E_{\mathrm{ph}}$. The model with generalized Fermi surface topologies is now extended to intermediate and strong coupling with the use of the McMillan effective approach to the Eliashberg equations. In this work, we use a momentum-independent pairing interaction, supposing that the superconducting order parameter has s-wave symmetry. We will evaluate some properties of the sulfur hydride superconductor, $\mathrm{H}_{3} \mathrm{~S}$, as the isotope mass exponent, $\alpha$, in terms of the coupling constant and the parameters of the model. The multiband scenario of superconductivity had previously been used to describe the isotope coefficient in other superconductor materials such as cuprate superconductors [43], hydrides [36, 44], and organic materials [45].

\section{Model and Calculation}

Next, we describe the schematic overlapping-band model which has been successfully used by us to describe properties, in particular the isotope mass exponent, of cuprate superconductors and iron-based oxypnictide superconductors $[40,42]$.

We begin with the gap equation

$$
\Delta\left(k^{\prime}\right)=\sum_{k} V\left(k, k^{\prime}\right) \Delta(k) \frac{\tanh \left(E_{k} / 2 k_{\mathrm{B}} T\right)}{2 E_{k}},
$$

with $V\left(k, k^{\prime}\right)$ the pairing interaction, $k_{\mathrm{B}}$ the Boltzmann constant, and $E_{k}^{2}=\varepsilon_{k}^{2}+\Delta_{k}^{2}$, where $\epsilon_{k}=\hbar^{2} k^{2} / 2 m$ are the selfconsistent, single-particle energies.

Then, for the electron-phonon interaction, we have considered $V\left(k, k^{\prime}\right)=V_{0}$, where $V_{0}$ is a constant if $\left|\epsilon_{k}\right| \leq E_{\mathrm{ph}}$ and $\left|\epsilon_{k}^{\prime}\right| \leq E_{\mathrm{ph}}$ and 0 elsewhere. $E_{\mathrm{ph}}$ is the electron-phonon coupling energy. As usual, the attractive BCS interaction is nonzero only for unoccupied orbitals in the neighborhood of the Fermi level, $E_{\mathrm{F}}$. The superconducting order parameter $\Delta(k)=\Delta(T)$ if $\left|\epsilon_{k}\right| \leq E_{\mathrm{ph}}$ and 0 elsewhere.

With these considerations, we propose a two-component model with overlapping bands. The generalized Fermi sea proposed here consists of concentric spherical shells separating occupied orbitals. We propose a Fermi surface with two bands as a prototype of band overlapping [46]. As a particular distribution in momentum space, the following form has been considered:

$$
n_{k}=\Theta\left(\gamma k_{\mathrm{F}}-k\right)+\Theta\left(\gamma k_{\mathrm{F}}-k\right) \Theta\left(k-\beta k_{\mathrm{F}}\right)
$$

with $k_{\mathrm{F}}$ the Fermi momentum and $0<\beta<\gamma<1$. The distribution in momentum induces another distribution in 
energy, $E_{\beta}<E_{\gamma}$, where $E_{\beta}=\beta^{2} E_{\mathrm{F}}$ and $E_{\gamma}=\gamma^{2} E_{\mathrm{F}}$ [47]. In order to keep the average number of electron states constant, the parameters in the system are related by the equation $2 \gamma^{2}-\beta^{2}=1$; then, only one of the relevant parameters is independent. We consider a high-frequency electron-optical-phonon coupling and require the band overlapping to be of order (or smaller) than the cutoff energy. That means $\left(1-\gamma^{2}\right) E_{\mathrm{F}} \leq E_{\mathrm{ph}}$. This can be rewritten as $1-\gamma^{2}=2 \nu \delta$, where $2 \nu=E_{\mathrm{ph}} / E_{\mathrm{F}}$ and $0<\delta<1$. The minimum $\gamma^{2}$ value consistent with our model is $\gamma_{\mathrm{ph}}^{2}=$ $1-\left(E_{\mathrm{ph}} / E_{\mathrm{F}}\right)$. While $E_{\mathrm{F}}-E_{\gamma} \leq E_{\mathrm{ph}}$, the energy difference between the normal and the anomalously occupied states must be provided by the material itself.

In the last framework, the summation in equation (1) is changed to an integration which is done over the (symmetric) generalized Fermi surface defined above. One gets

$$
\begin{aligned}
1= & \frac{V_{0} D(E)}{2} \int_{E_{\gamma}-E_{\mathrm{ph}}}^{E_{\gamma}+E_{\mathrm{ph}}} \tanh \left(\frac{\sqrt{\Xi_{k}}}{2 k_{\mathrm{B}} T}\right) \frac{d \varepsilon_{k}}{\sqrt{\Xi_{k}}}+\frac{V_{0} D(E)}{2} \\
& \int_{E_{\beta}}^{E_{\mathrm{F}}} \tanh \left(\frac{\sqrt{\Xi_{k}}}{2 k_{\mathrm{B}} T}\right) \frac{d \varepsilon_{k}}{\sqrt{\Xi_{k}}} .
\end{aligned}
$$

In this equation, $\Xi_{k}=\left(\epsilon_{k}-E_{\mathrm{F}}\right)^{2}+\Delta(T)^{2}, V_{0}$ is the effective attractive interaction of the BCS model, and $D(E)$ is the electronic density of states, which will be taken as a constant in the integration range.

The two integrals correspond to the bands proposed by equation (2). The integration over the surface at $E_{\gamma}$ in the first band is restricted to states in the interval $E_{\gamma}-E_{\mathrm{ph}} \leq$ $E_{k} \leq E_{\gamma}+E_{\mathrm{ph}}$. In the second band, in order to conserve particle number, the integration is restricted to the interval $E_{\beta} \leq E_{k} \leq E_{\mathrm{F}}$, if $E_{\gamma}+E_{\mathrm{ph}}>E_{\mathrm{F}}$, with $E_{\beta}=\left(2 \gamma^{2}-1\right) E_{\mathrm{F}}$, in terms of the parameter $\gamma$ in our model.

The critical temperature is introduced via equation (3) at $T=T_{c}$, where the gap becomes $\Delta\left(T_{c}\right)=0$. At this temperature, equation (3) is reduced to

$$
\begin{aligned}
1= & \frac{V_{0} D(E)}{2} \int_{E_{\gamma}-E_{\mathrm{ph}}}^{E_{\gamma}+E_{\mathrm{ph}}} \tanh \left(\frac{\epsilon_{k}-E_{F}}{2 k_{\mathrm{B}} T_{c}}\right) \frac{\mathrm{d} \epsilon_{k}}{\epsilon_{k}-E_{\mathrm{F}}}+\frac{V_{0} D(E)}{2} \\
& \int_{E_{\beta}}^{E_{\mathrm{F}}} \tanh \left(\frac{\epsilon_{k}-E_{\mathrm{F}}}{2 k_{\mathrm{B}} T_{c}}\right) \frac{\mathrm{d} \epsilon_{k}}{\epsilon_{k}-E_{\mathrm{F}}},
\end{aligned}
$$

which is to be numerically evaluated. Taking into account that $V_{0} D(E)=\left(\lambda-\mu^{*}\right) /(1+\lambda)$, where $\mu^{*}$ is the Coulomb pseudopotential, the last equation relates $T_{c}$ to the electron-phonon coupling parameter $\lambda$ and to the anomalous occupancy $\gamma^{2}$ parameter. This relationship determines the $\gamma^{2}$ values which reproduce the critical temperature of $\mathrm{H}_{3} \mathrm{~S}$ in the intermediate and strong coupling region.

At $T=0 \mathrm{~K}$, equation (3) will be evaluated and $\lambda$ values, consistent with the model, and which reproduce the zerotemperature superconducting gap ( $\Delta_{0}$ values), will be obtained:

$$
\begin{aligned}
1= & \frac{V_{0} D(E)}{2} \sinh ^{-1} \frac{E_{\mathrm{ph}}+\left(\gamma^{2}-1\right) E_{\mathrm{F}}}{\Delta_{0}}+\frac{V_{0} D(E)}{2} \sinh ^{-1} \\
& \frac{E_{\mathrm{ph}}+\left(1-\gamma^{2}\right) E_{F}}{\Delta_{0}}+\frac{V_{0} D(E)}{2} \sinh ^{-1} \frac{2\left(1-\gamma^{2}\right) E_{\mathrm{F}}}{\Delta_{0}} .
\end{aligned}
$$

Because theoretical results in the literature are quite different among themselves [48-50], the isotope exponent in the harmonic approximation, $\alpha$, is also evaluated. The twoband model in this work, along with the Cooper pair equation, is used in order to obtain $\alpha$. Strong and intermediate coupling effects are taken into account with the effective McMillan approximation which is valid for values of the coupling constant $\lambda<1.5$.

The isotope coefficient in the harmonic approximation is given by

$$
\alpha=-\frac{\mathrm{d} \ln T_{\mathrm{c}}}{\mathrm{d} \ln M} .
$$

Hence, we obtain

$$
\alpha=0.5\left[1+\delta \frac{2 Y-1+((\delta+2 Y-1) / D)}{\delta(1-2 Y)-1-D}\right],
$$

with $\delta=\left(1-\gamma^{2}\right) E_{\mathrm{F}} / E_{\mathrm{ph}}$, where

$$
D=\sqrt{\delta^{2}+2(2 Y-1) \delta+1},
$$

and $Y$ is given by

$$
Y=\exp \left[\frac{1.04(1+\lambda)}{\lambda-\mu^{*}(1+0.62 \lambda)}\right] .
$$

Equations (7)-(9) allow one to study the effect of intermediate and strong coupling up to $\lambda=1.5$ in our energy band overlapping model.

The model presented in this section can be useful to describe sulfur hydride superconductors. As was discussed in references [25, 47], tighter-bound Cooper pairs arise by generalizing the assumed spherical Fermi sea without invoking neither stronger electron-phonon coupling nor unconventional interaction mechanisms. Then, the range for the coupling parameter $0.4<\lambda<1.5$ in the intermediate and strong coupling regions, compatible with the McMillan approximation, is taken [32]. The $\lambda$ range could be extended up to $\lambda=2$ with the Allen-Dynes approximation [33,34].

Different values of $\mu^{*}$, as reported in the literature $[6,12,39]$, are used in our calculations. Overlapping parameter values $\gamma^{2}$, consistent with the model, are obtained for the material. The relationship between the characteristic parameters will be obtained for $\mathrm{H}_{3} \mathrm{~S}$ and used to evaluate the isotope mass exponent. The pairing in the superconducting state is taken as due, principally, to high-frequency optical modes.

\section{Results and Discussion}

In this section, we report the results for the properties of $\mathrm{H}_{3} \mathrm{~S}$ obtained with our model. Two extreme values of the Coulomb pseudopotential $\mu^{*}=0.1$ and $\mu^{*}=0.16$ are taken from 
references $[6,12,39]$. The high-energy stretching mode $E_{\mathrm{ph}}=$ $200 \mathrm{meV}$ is assumed to hold. The Migdal approximation, $E_{\mathrm{ph}} / E_{\mathrm{F}} \ll 1$, might not be satisfied when the multiband electronic structure of $\mathrm{H}_{3} \mathrm{~S}$ is taken into account. Then, the ratio $E_{\mathrm{ph}} / E_{\mathrm{F}}$ in the range $0.5 \geq E_{\mathrm{ph}} / E_{\mathrm{F}} \geq 2 / 9$, with $E_{\mathrm{ph}} / E_{\mathrm{F}}=$ $2 / 9$ as the threshold to the multiband region $[17,51]$, will be selected in our calculations.

In Figure 1, with the help of equation (4), the behavior of the coupling parameter $\lambda$, as a function of the band overlapping $\gamma^{2}$, is shown for $\mathrm{H}_{3} \mathrm{~S}$ at $150 \mathrm{Gpa}$. At this pressure, the critical temperature $T_{\mathrm{c}}$ is about $203 \mathrm{~K}$. We select $E_{\mathrm{ph}}=$ $200 \mathrm{meV}$. The values $\mu^{*}=0.1$ and $\mu^{*}=0.16$ are taken in the calculations, and the full line represents $\mu^{*}=0.1$, while the dashed one is for $\mu^{*}=0.16$. The Fermi energy satisfies $E_{\mathrm{ph}} / E_{\mathrm{F}}=0.222$. The range of $\lambda$ values obtained with our model is in the intermediate and strong coupling region $\lambda \leq 1.5$, where the McMillan approximation is valid. For a greater value of the Coulomb pseudopotential, a stronger electron-phonon coupling is required, as seen in this figure.

In Figure 2, the coupling parameter $\lambda$ as a function of the band overlapping $\gamma^{2}$ for $\mathrm{H}_{3} \mathrm{~S}$, at the critical temperature $T_{\mathrm{c}}=203 \mathrm{~K}$, is shown for two values of $E_{\mathrm{ph}}$. In the top curve $E_{\mathrm{ph}}=100 \mathrm{meV}$ and $E_{\mathrm{ph}} / E_{\mathrm{F}}=0.248$, and in the bottom one $E_{\mathrm{ph}}=200 \mathrm{meV}$ and $E_{\mathrm{ph}} / E_{\mathrm{F}}=0.285$. The Coulomb pseudopotential $\mu^{*}=0.1$ is satisfied in both cases. The $\lambda$ values obtained are within the intermediate coupling region.

In Figure 3, the curves for the zero-temperature gap, $\Delta_{0}$, consistent with those in the literature, are shown. The curves for $\Delta_{0}=60,40$, and $30 \mathrm{meV}$ are shown from top to bottom, the Coulomb pseudopotential was taken to be $\mu^{*}=0.1$, and $E_{\mathrm{ph}} / E_{\mathrm{F}}=0.4$. Comparison with other multicomponent models we observe that the upper curve in Figure 3 is compatible with the value of $\Delta_{0}=65 \mathrm{meV}$ with a multiband model in reference [36]. The middle curve in our figure is similar to $\Delta_{0}=43.8 \mathrm{meV}$, obtained with a two Fermi surface sheet model at $200 \mathrm{GPa}$ by Flores-Livas et al. [39]. These curves allow us to observe the behavior of the coupling constant in terms of the parameter $\gamma^{2}$ of our model. For a larger value of the gap, larger coupling constant values are required, but still consistent with the McMillan approximation.

Similar results are obtained by using different models. For example, with an analysis based on density-functional theory of electronic and vibrational properties of hydrogen and a Coulomb pseudopotential with $\mu^{*}=0.1$, they got a superconducting energy gap of $\Delta_{0}=62 \mathrm{meV}$ [52]. While in reference [18], they got a value of $\Delta_{0}=43 \mathrm{meV}$ with the phonon-mediated Eliashberg theory. On the other hand, in reference [53], a smaller value of $\Delta_{0}=28 \mathrm{meV}$ was obtained.

In Figure 4, the isotope mass exponent, $\alpha\left(\lambda, \gamma^{2}\right)$, for the value of the ratio $E_{\mathrm{ph}} / E_{\mathrm{F}}=0.40$, is evaluated via the relation $\delta=\left(\left(1-\gamma^{2}\right) E_{\mathrm{F}}\right) / E_{\mathrm{ph}}$ in equation (7). The curves for $\delta=0.03,0.06$, and 0.1 are shown from top to bottom, where $\mu^{*}=0.1$ in all the curves. With $\lambda>0.7$, we get $0.15<\alpha<0.37$, and these values are smaller but close to the BCS result, $\alpha=0.5$. At a given $\lambda$ value, the mass exponent is larger for smaller values of $\delta$. Our results for the isotope mass exponent agree with those obtained using other models in the

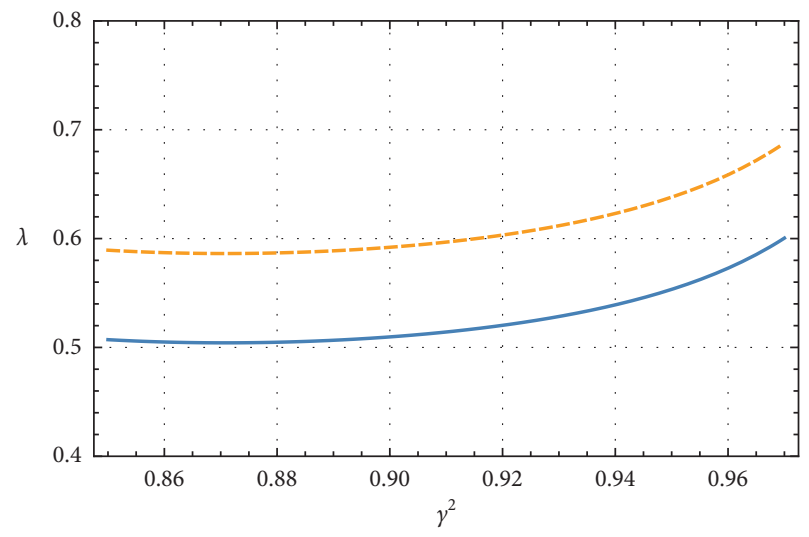

FIGURE 1: Values of the coupling constant, $\lambda$, in terms of the parameter $\gamma^{2}$ obtained in this work. The $T_{c}$ curves are given for the critical temperature of $203 \mathrm{~K}$. The Coulomb pseudopotential values $\mu^{*}=0.1$ (solid line) and $\mu^{*}=0.16$ (dashed line) are taken. The ratio $E_{\mathrm{ph}} / E_{\mathrm{F}}=0.222$.

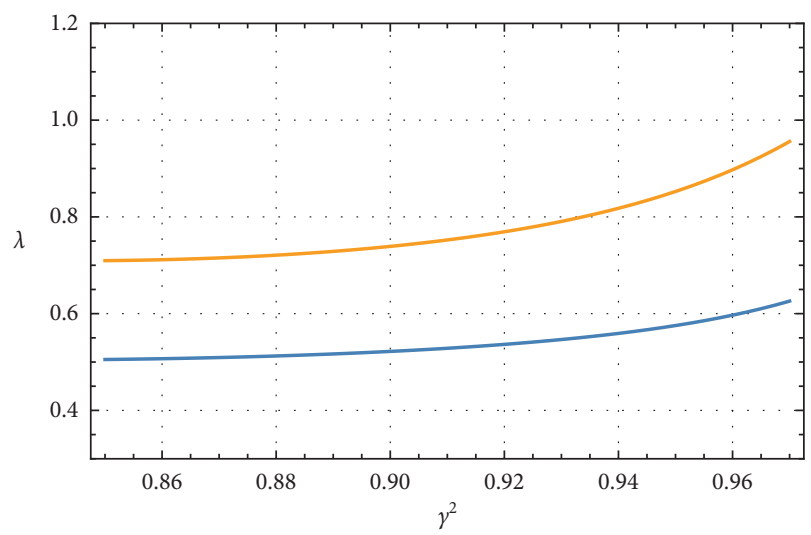

Figure 2: Values of the coupling constant, $\lambda$, in terms of the parameter $\gamma^{2}$ obtained in this work at $T_{\mathrm{c}}=203 \mathrm{~K}$. The Coulomb pseudopotential is $\mu^{*}=0.1$ for both curves; $E_{\mathrm{ph}}=200 \mathrm{meV}$ for the lower curve and $E_{\mathrm{ph}}=100 \mathrm{meV}$ for the upper one. $E_{\mathrm{ph}} / E_{\mathrm{F}}=0.285$ and 0.248 , respectively.

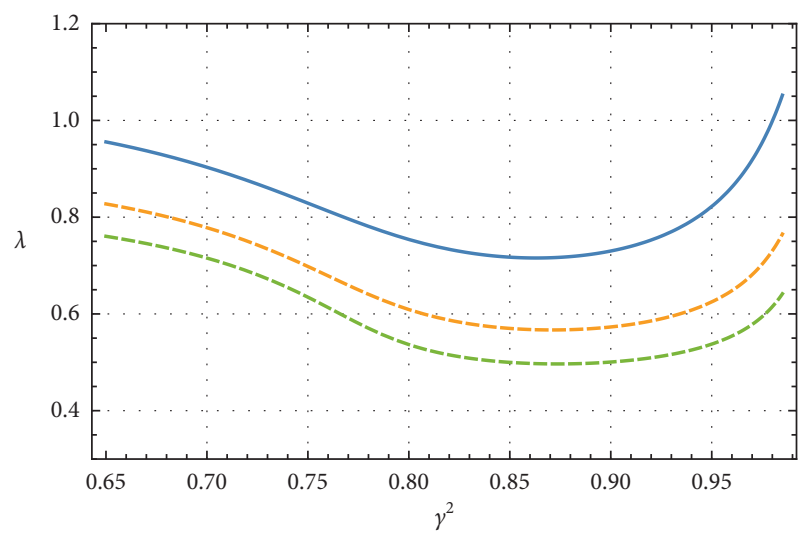

FIgURE 3: The curves for three values of the zero-temperature gap, $\Delta_{0}$, are shown; $\mu^{*}=0.1$ and $E_{\mathrm{ph}} / E_{\mathrm{F}}=0.40$. The shown curves are for $\Delta_{0}=60,40$, and $30 \mathrm{meV}$, from top to bottom. 


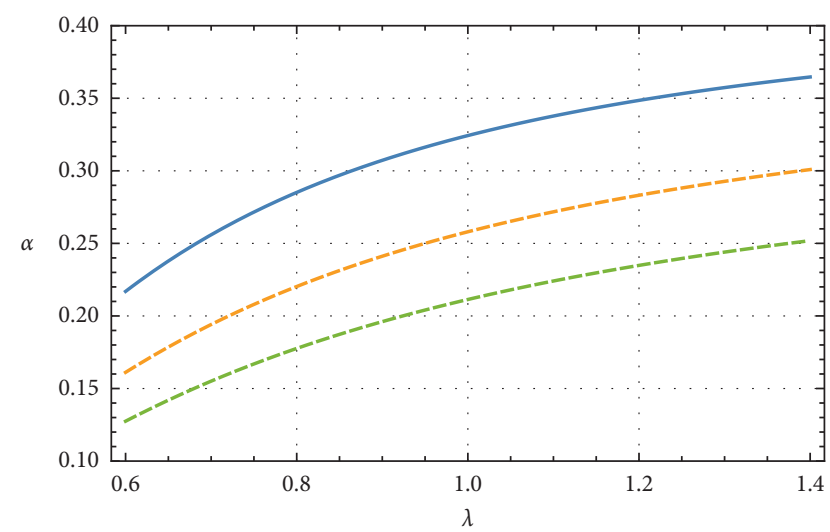

FIGURE 4: The isotope mass exponent, $\alpha$, is shown as a function of the coupling constant, $\lambda$, using $\delta$ values obtained with our model, where $\delta=\left(1-\gamma^{2}\right) E_{\mathrm{F}} / E_{\mathrm{ph}}$. The curves for $\delta=0.03,0.06$, and 0.1 are plotted from top to bottom.

literature, but are lower than the experimental value [50,54]. The $\alpha$ values obtained in this work are in the range of those in references $[6,18,54,55]$, evaluated with different models for $\mathrm{H}_{3} \mathrm{~S}$. As seen in the graphic, for greater $\lambda$ values, the $\alpha$ results do not change notoriously. The $\lambda$ range could be extended with the Allen-Dynes approximation up to $\lambda=2[33,34]$.

\section{Conclusions}

Based on the electronic structure for $\mathrm{H}_{3} \mathrm{~S}[36,39]$ and in order to increase the density of states at the Fermi level for high-temperature superconductors, we presented a twocomponent model with generalized Fermi surface topologies obtained via band overlapping. We considered two overlapping bands at the Fermi level, where the order parameter is taken to have s-wave symmetry. The band overlapping allowed for improvement on the results obtained with an $s$-wave mean-field approximation in a scheme in which the electron-phonon interaction is the relevant one for the high$T_{c}$ mechanism (clean limit). The energy scale of the band overlapping is of the order of the phonon energy, $\left(1-\gamma^{2}\right) E_{\mathrm{F}} \leq E_{\mathrm{ph}}$. A nonstandard electron-phonon coupling is considered, and the minimum value of the band overlapping parameter, $\gamma^{2}$, is consistent with this value. This energy is then the overall scale that determines the highest $T_{c}$ and gives consistency to the model because it requires an energy scale accessible to the lattice. We used this model to describe sulfur hydride superconductors, within the BCS framework, and the model was extended to include intermediate coupling with the McMillan approximation. As mentioned before, tighter-bound Cooper pairs arise by generalizing the assumed spherical Fermi sea without invoking neither stronger electron-phonon coupling nor unconventional interaction mechanisms. In fact, the $\lambda$ values obtained in this work for the critical temperature and the zero-temperature gap, $\Delta_{0}$, are in the intermediate coupling region. The $\mathrm{H}_{3} \mathrm{~S}$ isotope exponent values we calculated agree with those reported in the literature using other models, but are lower than the experimental value. We were not able to get a larger value of the isotope mass exponent. That suggests the necessity to introduce modifications in order to account for additional elements. However, this schematic model has the capability to generically describe some properties of the $\mathrm{H}_{3} \mathrm{~S}$ superconductor and can be a guide for a more detailed study of these materials with, for example, the Eliashberg theory.

\section{Data Availability}

The data used to support the findings of this work are included within the article.

\section{Conflicts of Interest}

The authors declare that there are no conflicts of interest regarding the publication of this paper.

\section{Acknowledgments}

It is a pleasure to thank S. Orozco, M. Moreno, and E. Moreno for their valued comments.

\section{References}

[1] A. P. Drosdov, M. I. Eremets, I. A. Troyan, V. Ksenofonton, and I. Shyling, "Conventional superconductivity at 203 kelvin at high pressures in the sulfur hydride system," Nature, vol. 525, no. 7567, pp. 73-76, 2015.

[2] A. P. Drosdov, M. I. Eremets, and I. A. Troyan, "Conventional superconductivity at $190 \mathrm{~K}$ at high pressures," 2014, https:// arxiv.org/abs/1412.0460.

[3] N. W. Ashcroft, "Hydrogen dominant metallic alloys: high temperature superconductors?," Physical Review Letters, vol. 92, no. 18, Article ID 187062, 2004.

[4] V. L. Ginzburg, "Superfluidity and superconductivity in the universe," Journal of Statistical Physics, vol. 1, no. 1, pp. 3-24, 1969.

[5] N. W. Ashcroft, "Metallic hydrogen: a high-temperature superconductor?," Physical Review Letters, vol. 21, no. 26, pp. 1748-1749, 1968.

[6] I. Errea, M. Calandra, C. Pickard et al., "High-pressure hydrogen sulfide from first principles: a strongly anharmonic phonon-mediated superconductor," Physical Review Letters, vol. 114, no. 15, Article ID 157004, 2015.

[7] M. Einaga, M. Sakata, T. Ishikawa et al., "Crystal structure of the superconducting phase of sulfur hydride," Nature Physics, vol. 12, no. 9, pp. 835-838, 2016.

[8] N. Bernstein, C. S. Hellberg, M. D. Johannes, I. I. Mazin, and M. J. Mehl, "What superconducts in sulfur hydrides under pressure and why," Physical Review B, vol. 91, no. 6, Article ID 060511, 2015.

[9] D. Duan, X. Huang, F. Tian et al., "Pressure induced decomposition of solid hydrogen sulfide," Physical Review B, vol. 91, no. 18, Article ID 180502, 2015.

[10] A. P. Durajski and R. Szcześniak, "Structural, electronic, vibrational, and superconducting properties of hydrogenated chlorine," The Journal of Chemical Physics, vol. 149, no. 7, Article ID 074101, 2018.

[11] M. Somayazulu, M. Ahart, A. K. Mishra et al., "Evidence for superconductivity above $260 \mathrm{~K}$ in lanthanum superhydride at megabar pressures," Physical Review Letters, vol. 122, no. 2, Article ID 027001, 2019. 
[12] D. Duan, Y. Liu, F. Tian et al., "Pressure-induced metallization of dense $\left(\mathrm{H}_{2} \mathrm{~S}\right)_{2} \mathrm{H}_{2}$ with high- $T_{\mathrm{c}}$ superconductivity," Nature Scientific Reports, vol. 4, no. 1, p. 6968, 2014.

[13] A. P. Durajsky, R. Szcze, śniak, and Y. Li, "Non-BCS thermodynamic properties of $\mathrm{H}_{2} \mathrm{~S}$ superconductor," Physica $C$ : Superconductivity and its Applications, vol. 515, pp. 1-6, 2015.

[14] Y. Li, J. Hao, H. Liu, Y. Li, and Y. Ma, "The metallization and superconductivity of dense hydrogen sulfide," The Journal of Chemical Physics, vol. 140, no. 17, Article ID 1747125, 2014.

[15] L. Gor'kov and V. Kresin, "Pressure and high- $T_{c}$ superconductivity in sulfur hydrides," Nature Letter, vol. 10, p. 14964, 2015.

[16] N. A. Kudryashov, A. A. Kutukov, and E. A. Mazur, "Normal state of metallic hydrogen sulfide," Physics of Metals and Metallography, vol. 118, no. 2, pp. 113-122, 2017.

[17] A. P. Durajsky and R. Szczésniak, "Migdal-Eliashberg equations-the effective model for superconducting state in $\mathrm{H}_{2} \mathrm{~S}$," 2016, https://arxiv.org/abs/1609.06079.

[18] A. P. Durajsky, R. Szczśniak, and L. Pietronero, "Hightemperature study of superconducting hydrogen and deuterium sulfide," Annals of Physics, vol. 528, no. 5, pp. 358-364, 2016.

[19] A. Bianconi and T. Jarlborg, "Lifshitz transitions and zero point lattice fluctuations in sulfur hydride showing near room temperature superconductivity," Novel Superconducting Materials, vol. 1, no. 1, p. 37, 2015.

[20] W. Sano, T. Korensune, T. Tadano, R. Akashi, and R. Arita, "Effect of van Hove singularities on high- $T_{\mathrm{c}}$ superconductivity in $\mathrm{H}_{3} \mathrm{~S}$," Physical Review B, vol. 93, no. 9, Article ID 094525, 2016.

[21] Y. Quan and W. E. Pickett, "Van-Hove singularities and spectral smearing in high-temperature superconducting $\mathrm{H}_{3} \mathrm{~S}$," Physical Review B, vol. 93, no. 10, Article ID 104526, 2016.

[22] R. Szczęśniak and A. P. Durajsky, "Unusual sulfur isotope effect and extremely high critical temperature in $\mathrm{H}_{3} \mathrm{~S}$ superconductor," Scientific Reports, vol. 8, no. 1, p. 6037, 2018.

[23] J. E. Hirsch and D. J. Scalapino, "Enhanced superconductivity in quasi two-dimensional systems," Physical Review Letters, vol. 56, no. 25, pp. 2732-2735, 1986.

[24] A. Nakanishi, T. Ishikawa, and K. Shimizu, "First-Principles study on superconductivity of P- and Cl-doped $\mathrm{H}_{3} \mathrm{~S}$," Journal of the Physical Society of Japan, vol. 87, no. 12, article 124711, 2018.

[25] G. Carmona, R. M. Méndez-Moreno, S. Orozco et al., "Anomalous fermionic occupancy for High- $T_{\mathrm{c}}$ superconducting layered materials," Modern Physics Letters B, vol. 6, no. 15, pp. 935-942, 1992.

[26] M. Moreno, R. M. Méndez-Moreno, M. A. Ortiz, and S. Orozco, "Energy band overlapping in high- $T_{c}$ superconductors," Modern Physics Letters B, vol. 10, no. 30, pp. 1483-1490, 1996.

[27] S. Orozco, R. M. Méndez-Moreno, and M. A. Ortiz, “The gap and the upper critical field $\mathrm{H}_{c 2}$ as function of doping for high$T_{c}$ cuprates," Advances in Condensed Matter Physics, vol. 2014, Article ID 212895, 7 pages, 2014.

[28] T. X. R. Souza and F. Marsiglio, "Systematic study of the superconducting critical temperature in two- and three-dimensional tigh-binding models: a possible scenario for superconducting $\mathrm{H}_{3} \mathrm{~S}$," Physical Review B, vol. 94, no. 18, Article ID 184509, 2016.

[29] E. F. Talantsev, W. P. Crump, J. G. Storey, and J. L. Tallon, "London penetration depth and thermal fluctuations in the sulphur hydride $203 \mathrm{~K}$ superconductor," Annalen der Physik, vol. 529, no. 3, Article ID 1600390, 2017.
[30] N. A. Kudryashov, A. A. Kutukov, and E. A. Mazur, "Metal hydrogen sulfide superconducting temperature," Novel Superconducting Materials, vol. 3, no. 1, pp. 1-5, 2017.

[31] F. Capitani, B. Langerome, J.-B. Brubach et al., "Spectroscopic evidence of a new energy scale for superconductivity in $\mathrm{H}_{3} \mathrm{~S}$," Nature Physics, vol. 13, no. 9, pp. 859-863, 2017.

[32] W. L. McMillan, "Transition temperature of strong-coupled superconductors," Physical Review, vol. 167, no. 2, pp. 331-344, 1968.

[33] R. C. Dynes, "McMillan's equation and the $T_{\mathrm{c}}$ of superconductors," Solid State Communications, vol. 10, no. 7, pp. 615-618, 1972.

[34] P. B. Allen and R. C. Dynes, "Transition temperature of strong-coupled superconductors reanalyzed," Physical Review B, vol. 12, no. 3, pp. 905-922, 1975.

[35] J. P. Carbotte, "Properties of boson-exchange superconductors," Reviews of Modern Physics, vol. 62, no. 4, pp. 1027-1157, 1990.

[36] T. Jarlborg and A. Bianconi, "Breakdown of the Migdal approximation at Lifshitz transitions with giant zero-point motion in the $\mathrm{H}_{3} \mathrm{~S}$ superconductor," Scientific Reports, vol. 6, no. 1, p. 24816, 2016.

[37] E. J. Nicol and J. P. Carbotte, "Comparison of pressurized sulfur hydride with conventional superconductors," Physical Review B, vol. 91, no. 22, Article ID 220507, 2015.

[38] A. Bianconi and T. Jarlborg, "Superconductivity above the lowest earth temperature in pressurized sulfur hydride," EPL (Europhysics Letters), vol. 112, no. 3, p. 37001, 2015.

[39] J. A. Flores-Livas, A. Sanna, and E. K. U. Gross, "High temperature superconductivity in sulfur and selenium hydrides at high pressure," The European Physical Journal B, vol. 89 , no. 3, p. 63, 2016.

[40] S. Orozco, M. A. Ortiz, R. M. Méndez-Moreno, and M. Moreno, "A model for the isotope effect in high- $T_{\mathrm{c}}$ superconductors," Physica C: Superconductivity, vol. 408-410, pp. 346-347, 2004.

[41] S. Orozco, R. M. Méndez-Moreno, M. A. Ortiz, and M. Moreno, "The charge carrier density effect on the superconductor parameters in a two-band model," Physica C: Superconductivity, vol. 449, no. 1, pp. 67-72, 2006.

[42] S. Orozco, M. A. Ortiz, R. M. Méndez-Moreno, and G. Murguía, "A multiband model for $\mathrm{SmFeAsO}_{1-\mathrm{x}} \mathrm{F}_{\mathrm{x}}$," Journal of Physics: Conference Series, vol. 400, no. 2, 2012.

[43] A. Perali, D. Innocenti, A. Valletta, and A. Bianconi, "Anomalous isotope effect near a 2.5 Lifshitz transition in a multi-band multi-condensate superconductor made of a superlattice of stripes," Superconductor Science and Technology, vol. 25, no. 12, 2012.

[44] A. Bussmann-Holder, J. Köhler, A. Simon, M.-H. Whangbo, A. Bianconi, and A. Perali, "The road map toward roomtemperature superconductivity: manipulating different pairing channels in systems composed of multiple electronic components," Condensed Matter, vol. 2, no. 3, p. 24, 2017.

[45] M. V. Mazziotti, A. Valletta, G. Campi, D. Innocenti, A. Perali, and A. Bianconi, "Possible Fano resonance for high- $T_{\mathrm{c}}$ multigap superconductivity in p-terphenyl doped by $\mathrm{K}$ at the Lifshitz transition," EPL (Europhysics Letters ), vol. 118, no. 3, p. $37003,2017$.

[46] G. Murguía-Romero, S. Orozco, M. D. L. Á. Ortiz, R. M. Méndez-Moreno, and P. de la Mora, "A multiband model for $\mathrm{LaO}_{1-x} \mathrm{~F}_{x} \mathrm{FeAs}$," Advances in Science and Technology, vol. 75, pp. 167-172, 2010. 
[47] M. de Llano and J. P. Vary, "The Cooper pair problem for generalized Fermi surfaces," Journal of Physics: Condensed Matter, vol. 3, no. 3, pp. 329-335, 1991.

[48] D. R. Harshman and A. Fiory, "Compressed $\mathrm{H}_{3} \mathrm{~S}$ : intersublattice coulomb coupling in a high- $T_{\mathrm{c}}$ superconductor," Journal of Phys: Condensed Matter, vol. 29, no. 44, Article ID 445702, 2017.

[49] D. R. Harshman and A. Fiory, "On the isotope effect in compressed superconducting $\mathrm{H}_{3} \mathrm{~S}$ and $\mathrm{D}_{3} \mathrm{~S}$," Superconductor Science and Technology, vol. 30, no. 4, Article ID 045011, 2017.

[50] J. E. Hirsch and F. Marsiglio, "Hole superconductivity in $\mathrm{H}_{2} \mathrm{~S}$ and other sulfides under high pressure," Physica C: Superconductivity and its Applications, vol. 511, pp. 45-49, 2015.

[51] P. Baňacký, "On the mechanism of high-temperature superconductivity in hydrogen sulfide at $200 \mathrm{GPa}$ : transition into superconducting anti-adiabatic state in coupling to H-vibrations," Results in Physics, vol. 6, pp. 1-2, 2016.

[52] M. Borinaga, I. Errea, M. Calandra, F. Mauri, and A. Bergara, "Anharmonic effects in atomic hydrogen: superconductivity and lattice dynamical stability," Physical Review B, vol. 93, no. 17, 2016.

[53] E. F. Talantsev, "Classifying superconductivity in compressed $\mathrm{H}_{3}$ S.," 2019, https://arxiv.org/abs/1902.01772.

[54] R. Akashi, M. Kawamura, S. Tsuneyuki, Y. Nomura, and R. Arita, "First-principles study of the pressure and crystalstructure dependences of superconducting transition temperature in compressed sulfur hydrides," Physical Review B, vol. 91, no. 22, 2015.

[55] L. P. Gor'kov and V. Z. Kresin, "Colloquium: high pressure and road to room temperature superconductivity," Reviews of Modern Physics, vol. 90, no. 1, 2018. 

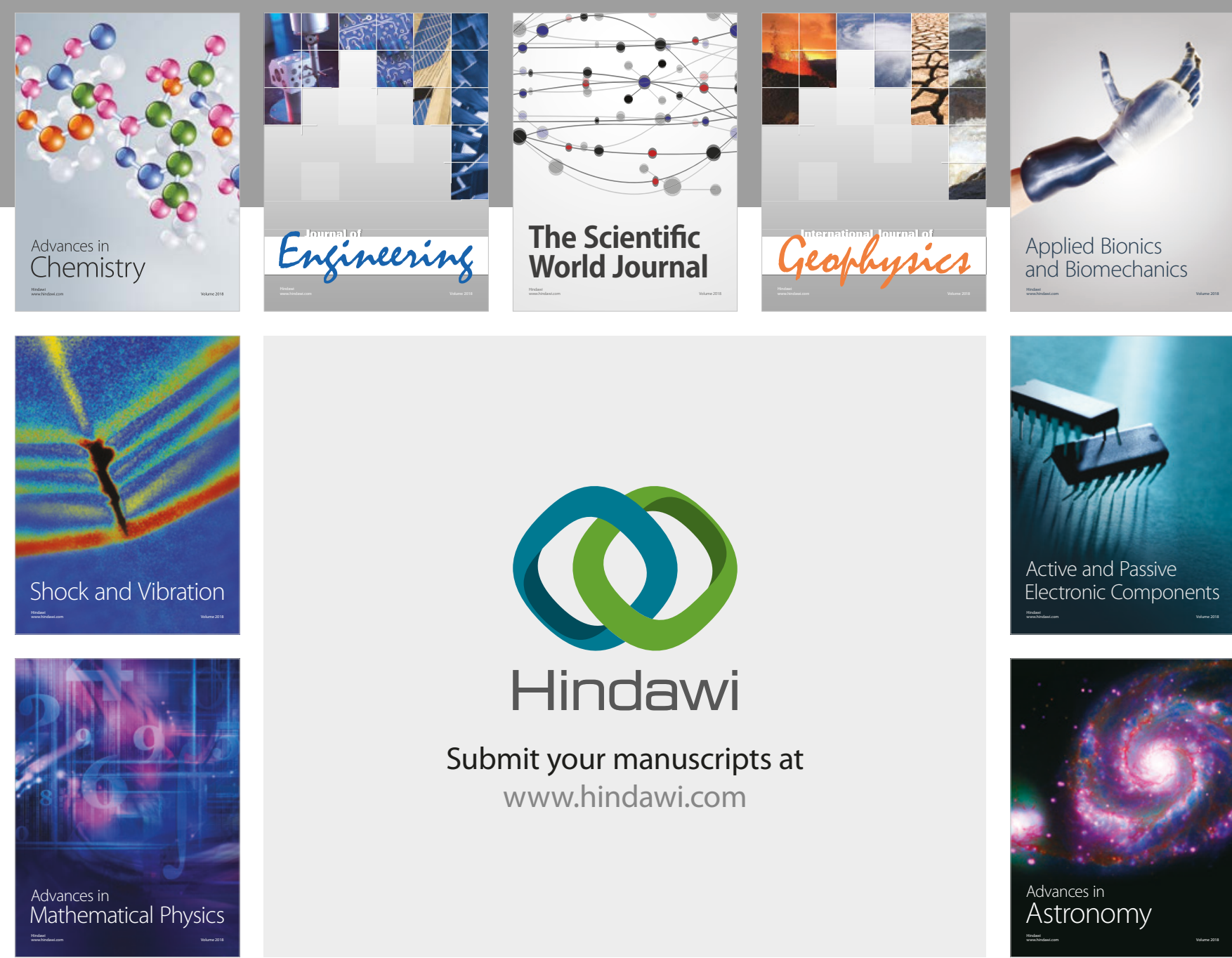

Submit your manuscripts at

www.hindawi.com

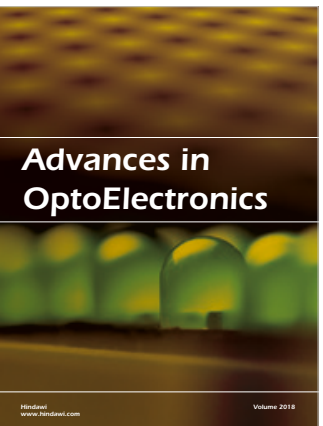

\section{Rotcting Machinery}
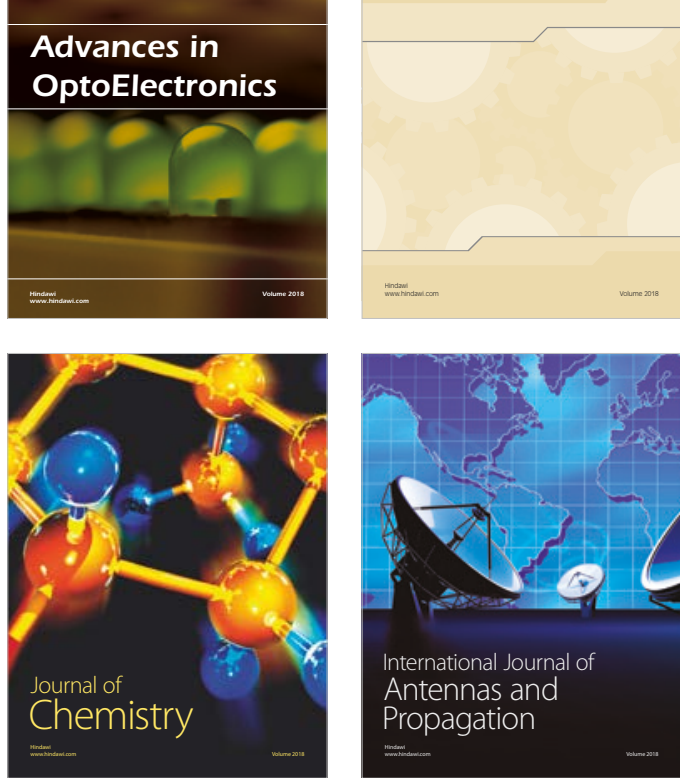

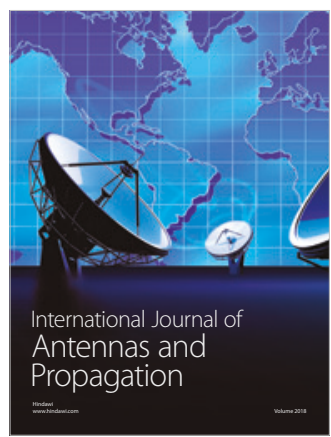

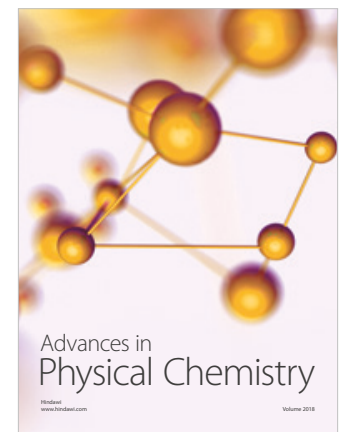

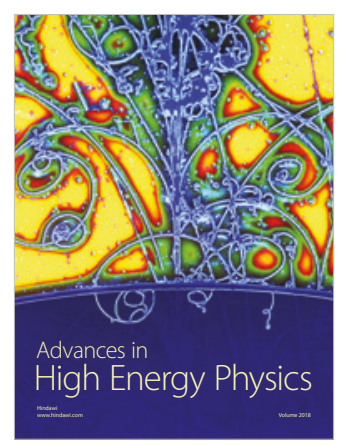

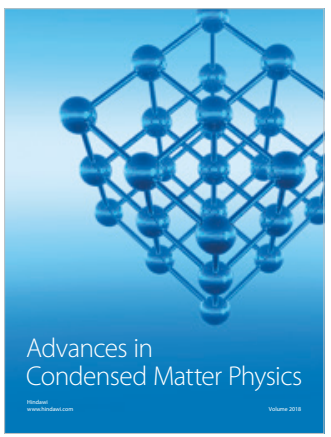

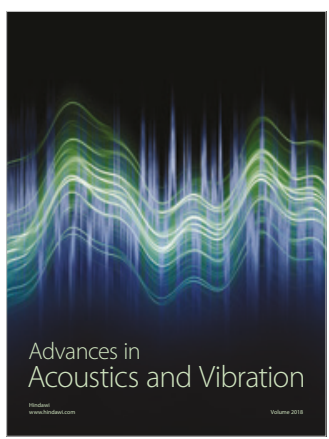

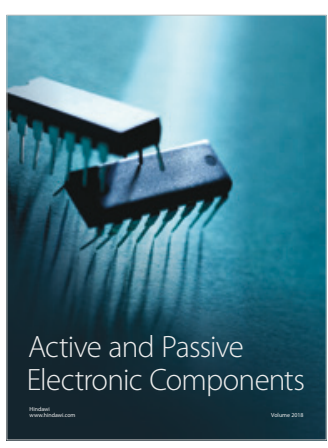
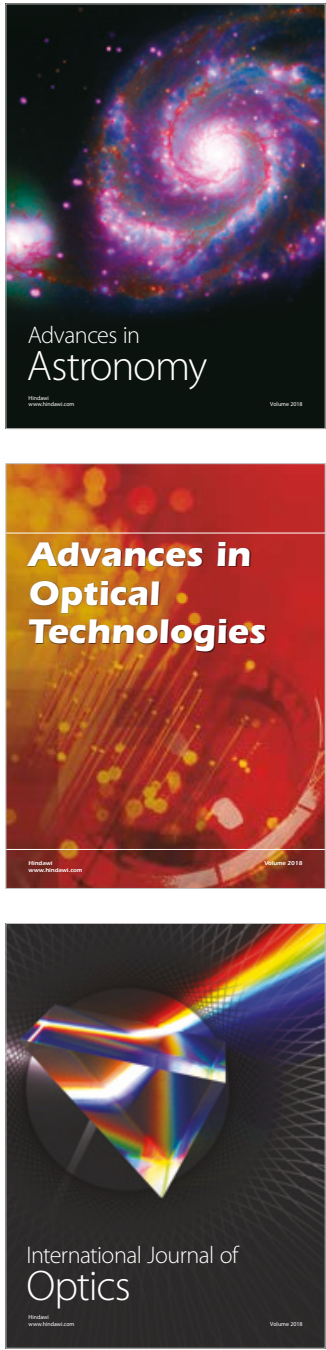